\title{
Clinical, Histological and Immunohistochemical Changes in Hypopigmented Mycosis Fungoides in Response to Narrow-Band UVB Phototherapy
}

Valeria Mateeva* and Miroslava Kadurina

Military Medical Academy Sofia, Bulgaria

\section{Case}

A 26- year old Caucasian woman of Bulgarian descent presented at our department with multiple disseminated hypopigmented macules on the trunk and extremities since more than 4 years (Figure 1A). The differential diagnosis included: vitiligo, pityriasis vesicolor and Mycosis fungoides- hypopigmented type. A punch biopsy was performed and histological examination revealed exocytosis of atypical lymphocytes with larger hyperchromic nuclei (Figure 1B). A punch biopsy from normally looking skin was taken and the histological and immunohistochemical examination showed pigment incontinence and epidermotropisme of single atypical, CD3+CD4+ lymphocytes. Clinical
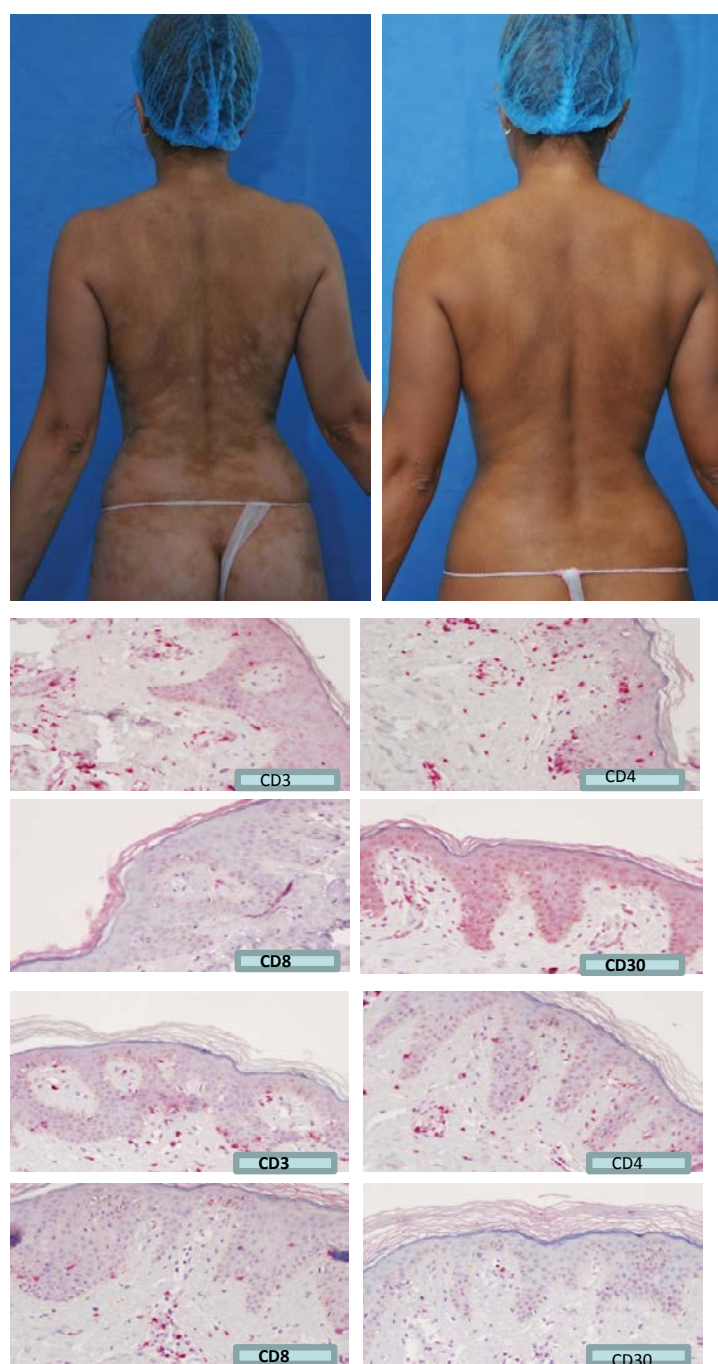

Figure 1: Clinical and histological response and change in immunohistochemical expression pattern following 22 procedures Narrowband UVN phototherapyA-B: clinical and histological picture before treatment; C-D: complete clinical response following 22 procedures Narrow-band UVB phototherapy and partial histological response with reduction of the CD3CD4-positive epidermotropic lymphocytes. and histological diagnosis of Mycosis fungoides- hypopigmented variant has been established.

A Narrow Band Ultraviolet B- Treatment (NB- UVB) has been initiated according to the phototherapy protocol at our clinic with 4 sessions/ weekly with initial dose of $0.3 \mathrm{~J} / \mathrm{cm}^{2}$ and incensement of the dose with 0,05 at each session. A total number of 27 sessions have been performed and complete clinical response (disappearance of all existing lesions for at least one month) has been achieved after the $22^{\text {st }}$ session (Figure 1C). Total UV dose was $17.5 \mathrm{~J} / \mathrm{cm}^{2}$ and complete clinical response was achieved at a dose of $11.85 \mathrm{~J} / \mathrm{cm}^{2}$. No maintenance phototherapy has been administered according to the protocol.

Skin biopsy after the end of the treatment taken from clinically cleared past lesion revealed fewer than initially, but still present, CD3+ $\mathrm{T}$ - cells, mostly CD4+, with large atypical hyperchromic nuclei in the epidermis (Figure 1D). Exocytosis of atypical lymphocytes with larger hyperchromic nuclei were still present in fewer numbers than initially, marking a partial histological response to the therapy.

The disease- free interval was 3,5 months. The patient presented with multiple hypopigmented lesions on her tights.

\section{Discussion}

The hypopigmented variant of Mycosis fungoides is rare among Caucasians and data about the efficacy and safety of NB- UVB treatment are scarce. The lower cumulative dose $\left(17.5 \mathrm{~J} / \mathrm{cm}^{2}\right)$ and the rapid complete response to treatment (after 22 sessions, or after a dose of $11.85 \mathrm{~J} / \mathrm{cm}^{2}$ ) in comparison to previously published studies in the literature (from 27.1- $59.6 \mathrm{~J} / \mathrm{cm}^{2}$ ) [1]. Another study revealed even higher number of sessions required for complete remission of hypopigmented mycosis fungoides in Asian patients (average 40 sessions) [2]. This significant difference could possibly be associated with the Caucasian origin of the patient and the flare skin type III according to Fitzpatrick, which could be sensitive to lower doses UVB in comparison to the Asian population with hypopigmented mycosis fungoides.

The phototherapy protocol we used differs from the currently described protocol in Asians [1]. The more often (4 times/ weekly compared to the previously published twice/ weekly) regiment could offer more rapid remission at a lower cumulative UV- dose. Nevertheless the short remission time ( 3.5 months) compared to a previously reported (2 months 6 years) disease- free period suggests

*Corresponding author: Valeria Mateeva, 3, Gueorgui Sofiiski, 1606 Sofia, Bulgaria, Tel: +359898631210, E-mail: vali_mateeva@hotmail.com

Received February 23, 2015; Accepted February 28, 2015; Published March 03 2015

Citation: Mateeva V, Kadurina M (2015) Clinical, Histological and Immunohistochemical Changes in Hypopigmented Mycosis Fungoides in Response to Narrow-Band UVB Phototherapy. Pigmentary Disorders 2: 167. doi:10.4172/2376-0427.1000167

Copyright: ( $) 2015$ Mateeva V, et al. This is an open-access article distributed under the terms of the Creative Commons Attribution License, which permits unrestricted use, distribution, and reproduction in any medium, provided the original author and source are credited. 
the importance of continuation of the therapy after complete clinical remission [1].

We also observed a lack of correlation between the complete clinical response and the histopathological findings in contrary to a previously published observation that clinical remission corresponds to histological remission in the patch stage mycosis fungoides [3]. This fact suggests a possible need of longer UVB- therapy after clinical remission in hypopigmented mycosis fungoides, since histological remission does not correlate with the clinical clearance of the disease and residual MF cells could provoke a clinical relapse and shorted disease- free period, a point that was not covered in the original article.

\section{Conclusion}

We presented a case of complete clinical remission of a patient with hypopigmented Mycosis fungoides with a significantly lower cumulative dose of NB- UVB $\left(17.5 \mathrm{~J} / \mathrm{cm}^{2}\right)$ with a different 4 times/ weekly regiment. Although there was complete clinical remission, histological remission was not achieved. We suggest that further investigations over the phototherapy protocols for hypopigmented Mycosis fungoides are needed, including a clinic- pathological correlation to assess the efficacy of the treatment. We suggest evaluation of the clinical and histological response and immunohistochemical expression to evaluate the therapeutic effect and eventually predict the length of the disease-free period.

\section{References}

1. Wongpraparut $C$, Setabutra $P$ (2012) Phototherapy for hypopigmented mycosis fungoides in Asians. Photodermatol Photoimmunol Photomed 28: 181-186.

2. Kanokrungsee S, Rajatanavin N, Rutnin S, Vachiramon V (2012) Efficacy of narrowband ultraviolet $B$ twice weekly for hypopigmented mycosis fungoides in Asians. Clin Exp Dermatol 37: 149-152.

3. Gökdemir G, Barutcuoglu B, Sakiz D, Köşlü A (2006) Narrowband UVB phototherapy for early-stage mycosis fungoides: evaluation of clinical and histopathological changes. J Eur Acad Dermatol Venereol 20: 804-809. 